DISTA-UPO/08

\title{
Star Product Geometries
}

\author{
Paolo Aschieri \\ Centro Studi e Ricerche "Enrico Fermi" Compendio Viminale, 00184 Roma, Italy \\ and Dipartimento di Scienze e Tecnologie Avanzate, Università del Piemonte Orientale, \\ and INFN, Sezione di Torino, gruppo collegato di Alessandria \\ aschieri@to.infn.it
}

\begin{abstract}
We consider noncommutative geometries obtained from a triangular Drinfeld twist. This allows to construct and study a wide class of noncommutative manifolds and their deformed Lie algebras of infinitesimal diffeomorphisms. This way symmetry principles can be implemented. We review two main examples [15]-18]: a) general covariance in noncommutative spacetime. This leads to a noncommutative gravity theory. b) Symplectomorphims of the algebra of observables associated to a noncommutative configuration space. This leads to a geometric formulation of quantization on noncommutative spacetime, i.e., we establish a noncommutative correspondence principle from $\star$-Poisson brackets to $\star$-commutators.

New results concerning noncommutative gravity include the Cartan structural equations for the torsion and curvature tensors, and the associated Bianchi identities. Concerning scalar field theories the deformed algebra of classical and quantum observables has been understood in terms of a twist $\widehat{\mathscr{F}}$ within the algebra.
\end{abstract}




\section{Introduction}

An interesting and promising field of research is the issue of spacetime structure in extremal energy regimes. There are evidences from General Relativity, string theory and black hole physics which support the hypothesis of a noncommutative structure. The simplest and probably most suggestive argument which points at a failure of the classical spacetime picture at high energy scales comes from the attempt of conjugating the principles of Quantum Mechanics with those of General Relativity (see [1], and for a review [2]). If one tries to locate an event with a spatial accuracy comparable with the Planck length $\left(\sim 10^{-33} \mathrm{~cm}\right)$, spacetime uncertainty relations necessarily emerge. In total analogy with Quantum Mechanics, uncertainty relations are naturally implied by the presence of noncommuting coordinates, $\left[x^{\mu}, x^{\nu}\right] \neq 0$. Therefore, below Planck length the usual description of spacetime as a pseudo-Riemannian manifold locally modeled on Minkowski space is not adequate anymore, and it has been proposed that it be described by a Noncommutative Geometry [3, 4, 5]. This line of thought has been pursued since the early days of Quantum Mechanics [6].

In this context two relevant issues are the formulation of General Relativity and the quantization of field theories on noncommutative spacetime. We address these issues by developing a differential geometry on noncommutative spacetime and phase space.

There are many noncommutative differential geometries (Connes type, quantum groups like, matrix or fuzzy,...). We work in the deformation quantization context; noncommutativity is obtained by introducing a formal $\star$-product on the algebra of smooth functions. The most widely studied form of noncommutativity is the Moyal-Weyl one (on spacetime $\left.\mathbb{R}^{4}\right), x^{\mu} \star x^{\nu}-x^{\nu} \star x^{\mu}=\mathrm{i} \theta^{\mu \nu}$ with $\theta^{\mu \nu}$ a constant antisymmetric matrix. This noncommutativity is obtained trought the $\star$-product $(f \star h)(x)=\left.\exp \left(\frac{\mathrm{i}}{2} \theta^{\mu \nu} \frac{\partial}{\partial x^{\mu}} \frac{\partial}{\partial y^{\nu}}\right) f(x) h(y)\right|_{x=y}$.

We focus on a class of star products obtained via a triangular Drinfeld twist [7]. This is a quite wide class (the examples in Section 3 include quantization of symplectic and also of Poisson structures). The algebra of functions of the noncommutative torus, of the noncommutative spheres [8] and of further noncommutative manifolds (so-called isospectral deformations) considered in [8], and in [9], [10], is associated to a $\star$-product structure obtained via a triangular Drinfeld twist (see [12] and, for the four-sphere in [8], see [11], [13]). The star products we study are however not the most general ones, in particular they are a subclass of those associated with a quasitriangular structure [14]: on that noncommutative algebra of functions there is an action of the braid group, in the case we study there is an action of the permutation group.

It is remarkable how far in the program of formulating a noncommutative differential geometry one can go using triangular Drinfeld twists. The study of this class of $\star$-products geometries are first examples that can uncover some common features of a wider class of 
noncommutative geometries.

In Section 2 we introduce the twist $\mathcal{F}$ and, starting from the principle that every product, and in general every bilinear map, is consistently deformed by composing it with the appropriate realization of the twist $\mathcal{F}$, we review the construction of noncommutative differential geometry as in [16, 15, 17]. A key point is that vectorfields have a natural $\star$-action ( $\star$-Lie derivative) on the noncommutative algebras of functions and tensorfields, giving rise to the concept of deformed derivations. These $\star$-derivations form a quantum Lie algebra ( $\star$-Lie algebra). In this way we consider the $\star$-Lie algebra of vectorfields. Next we can define covariant derivatives along vectorfields because their deformed Leibniz rule is the same as for Lie derivatives along vectorfields and because covariant derivative and Lie derivative coincide on functions. New results is this section include the Cartan structural equations for the torsion and curvature tensors, and the associated Bianchi identities.

In Section 3, following and developing the results of [18] we consider a noncommutative manifold $M$ (described by a twist $\mathcal{F}$ and an associated $\star$-product) with an extra Poisson structure $\Lambda$. If the noncommutative and the Poisson structures are compatible (in particular if the Poisson structure $\Lambda$ and the Poisson structure arising from the semiclassical limit of the $\star$-product are compatible) then the Poisson algebra of functions on $M$ can be deformed in a noncommutative $\star$-Poisson algebra. Otherwise stated the algebra of observables becomes noncommutative and equipped with a compatible $\star$-Lie algebra structure. Correspondingly the $\star$-Lie algebra of Hamiltonian vectorfields is a subalgebra of the $\star$-Lie algebra of vectorfields.

In Section 4 we apply this general construction to the infinite dimensional phase space of a scalar field theory. Here phase space has the usual Poisson structure $\{\Phi(x), \Pi(y)\}=$ $\delta(x-y)$, it also has a noncommutative structure $(\mathcal{F}$ and its $\star$-product $)$ induced from spacetime noncommutativity. Usual $\hbar$-quantization of this phase space can also be deformed with the twist $\mathcal{F}$ ( $\star$-deformed). We thus construct a $\star$-deformation of the algebra and Lie algebra of quantum observables of a scalar field. Starting from the usual canonical quanti-

zation map for field theories on commutative spacetime, $\Phi \stackrel{\hbar}{\rightarrow} \hat{\Phi}$, we thus uniquely obtain [18] a quantization scheme for field theories on noncommutative spacetime, and show that it satisfies a correspondence principle between $\star$-Poisson brackets and $\star$-commutators.

\section{Deformation by twists}

In this section we describe the general setting used to introduce a star product via a twist.

Consider a Lie algebra $g$ over $\mathbb{C}$, and its associated universal enveloping algebra $U g$. We recall that the elements of $U g$ are the complex numbers $\mathbb{C}$ and sums of products of elements $t \in g$, where we identify $t t^{\prime}-t^{\prime} t$ with the Lie algebra element $\left[t, t^{\prime}\right] . U g$ is an 
associative algebra with unit. It is a Hopf algebra with coproduct $\Delta: U g \rightarrow U g \otimes U g$, counit $\varepsilon: U g \rightarrow \mathbb{C}$ and antipode $S$ given on the generators as:

$$
\begin{array}{ll}
\Delta(t)=t \otimes 1+1 \otimes t & \Delta(1)=1 \otimes 1 \\
\varepsilon(t)=0 & \varepsilon(1)=1 \\
S(t)=-t & S(1)=1
\end{array}
$$

and extended to all $U(g)$ by requiring $\Delta$ and $\varepsilon$ to be linear and multiplicative (e.g. $\left.\Delta\left(t t^{\prime}\right):=\Delta(t) \Delta\left(t^{\prime}\right)=t t^{\prime} \otimes 1+t \otimes t^{\prime}+t^{\prime} \otimes t+1 \otimes t t^{\prime}\right)$, while $S$ is linear and antimultiplicative. In the sequel we use Sweedler coproduct notation

$$
\Delta(\xi)=\xi_{1} \otimes \xi_{2}
$$

where $\xi \in U g, \xi_{1} \otimes \xi_{2} \in U g \otimes U g$ and a sum over $\xi_{1}$ and $\xi_{2}$ is understood.

We extend the notion of enveloping algebra to formal power series in $\lambda$ (we replace the field $\mathbb{C}$ with the ring $\mathbb{C}[[\lambda]])$ and we correspondingly consider the Hopf algebra $(U g[[\lambda]], \cdot, \Delta, S, \varepsilon)$. In the sequel for sake of brevity we will often denote $U g[[\lambda]]$ by $U g$.

A twist $\mathcal{F}$ is an element $\mathcal{F} \in U g[[\lambda]] \otimes U g[[\lambda]]$ that is invertible and that satisfies

$$
\begin{gathered}
\mathcal{F}_{12}(\Delta \otimes i d) \mathcal{F}=\mathcal{F}_{23}(i d \otimes \Delta) \mathcal{F}, \\
(\varepsilon \otimes i d) \mathcal{F}=1=(i d \otimes \varepsilon) \mathcal{F},
\end{gathered}
$$

where $\mathcal{F}_{12}=\mathcal{F} \otimes 1$ and $\mathcal{F}_{23}=1 \otimes \mathcal{F}$.

In our context we in addition require*

$$
\mathcal{F}=1 \otimes 1+\mathcal{O}(\lambda)
$$

Property (2.5) states that $\mathcal{F}$ is a two cocycle, and it will turn out to be responsible for the associativity of the $\star$-products to be defined. Property (2.6) is just a normalization condition. From (2.7) it follows that $\mathcal{F}$ can be formally inverted as a power series in $\lambda$. It also shows that the geometry we are going to construct has the nature of a deformation, i.e. in the 0 -th order in $\lambda$ we recover the usual undeformed geometry.

We shall frequently use the notation (sum over $\alpha$ understood)

$$
\mathcal{F}=\mathrm{f}^{\alpha} \otimes \mathrm{f}_{\alpha} \quad, \quad \mathcal{F}^{-1}=\overline{\mathrm{f}}^{\alpha} \otimes \overline{\mathrm{f}}_{\alpha} .
$$

The elements $\mathrm{f}^{\alpha}, \mathrm{f}_{\alpha}, \overline{\mathrm{f}}^{\alpha}, \overline{\mathrm{f}}_{\alpha}$ live in $U g$.

${ }^{*}$ Actually it is possible to show that (2.7) is a consequence of (2.5), (2.6) and of $\mathcal{F}$ being at each order in $\lambda$ a finite sum of finite products of Lie algebra elements 
In order to get familiar with this notation we rewrite equation (2.5) and its inverse,

$$
\left((\Delta \otimes i d) \mathcal{F}^{-1}\right) \mathcal{F}_{12}^{-1}=\left((i d \otimes \Delta) \mathcal{F}^{-1}\right) \mathcal{F}_{23}^{-1},
$$

as well as (2.6) and (2.7) using the notation (2.8), explicitly

$$
\begin{aligned}
\mathrm{f}^{\beta} \mathrm{f}_{1}^{\alpha} \otimes \mathrm{f}_{\beta} \mathrm{f}_{2}^{\alpha} \otimes \mathrm{f}_{\alpha} & =\mathrm{f}^{\alpha} \otimes \mathrm{f}^{\beta} \mathrm{f}_{\alpha 1} \otimes \mathrm{f}_{\beta} \mathrm{f}_{\alpha_{2}}, \\
\overline{\mathrm{f}}_{1}^{\alpha} \overline{\mathrm{f}}^{\beta} \otimes \overline{\mathrm{f}}_{2}^{\alpha} \overline{\mathrm{f}}_{\beta} \otimes \overline{\mathrm{f}}_{\alpha} & =\overline{\mathrm{f}}^{\alpha} \otimes \overline{\mathrm{f}}_{\alpha_{1}} \overline{\mathrm{f}}^{\beta} \otimes \overline{\mathrm{f}}_{\alpha_{2}} \overline{\mathrm{f}}_{\beta}, \\
\varepsilon\left(\mathrm{f}^{\alpha}\right) \mathrm{f}_{\alpha} & =1=\mathrm{f}^{\alpha} \varepsilon\left(\mathrm{f}_{\alpha}\right), \\
\mathcal{F}=\mathrm{f}^{\alpha} \otimes \mathrm{f}_{\alpha} & =1 \otimes 1+\mathcal{O}(\lambda) .
\end{aligned}
$$

Consider now an algebra $A$ (over $\mathbb{C}[[\lambda]$ ), and an action of the Lie algebra $g$ on $A$, $a \mapsto t(a)$ where $t \in g$ and $a \in A$. We require compatibility of this action with the product in $A$ i.e. for any $t \in g$ we have a derivation of $A$,

$$
t(a b)=t(a) b+a t(b) .
$$

The action of $g$ on $A$ induces an action of the universal enveloping algebra $U g$ on $A$ (for example the element $t t^{\prime} \in U g$ has action $\left.t\left(t^{\prime}(a)\right)\right)$. We say that $A$ is a $U g$-module algebra, i.e., the algebra structure of the $U g$-module $A$ is compatible with the $U g$ action, for all $\xi \in U \Xi$ and $a, b \in A$,

$$
\xi(a b)=\mu \circ \Delta(\xi)(a \otimes b)=\xi_{1}(a) \xi_{2}(b) \quad, \quad \xi(1)=\varepsilon(\xi) 1 .
$$

(where 1 is the unit in $A$ ). This property is equivalent to (2.14).

Given a twist $\mathcal{F} \in U g \otimes U g$, we can construct a deformed algebra $A_{\star}$. The algebra $A_{\star}$ has the same vector space structure as $A$. The product in $A_{\star}$ is defined by

$$
a \star b=\mu \circ \mathcal{F}^{-1}(a \otimes b)=\overline{\mathrm{f}}^{\alpha}(a) \overline{\mathrm{f}}_{\alpha}(b) .
$$

In order to prove associativity of the new product we use (2.11) and compute:

$$
\begin{aligned}
(a \star b) \star c & =\overline{\mathrm{f}}^{\alpha}\left(\overline{\mathrm{f}}^{\beta}(a) \overline{\mathrm{f}}_{\beta}(b)\right) \overline{\mathrm{f}}_{\alpha}(c)=\left(\overline{\mathrm{f}}_{1}^{\alpha} \overline{\mathrm{f}}^{\beta}\right)(a)\left(\overline{\mathrm{f}}_{2}^{\alpha} \overline{\mathrm{f}}_{\beta}\right)(b) \overline{\mathrm{f}}_{\alpha}(c)=\overline{\mathrm{f}}^{\alpha}(a)\left(\overline{\mathrm{f}}_{\alpha_{1}} \overline{\mathrm{f}}^{\beta}\right)(b)\left(\overline{\mathrm{f}}_{\alpha_{2}} \overline{\mathrm{f}}_{\beta}\right)(c) \\
& =\overline{\mathrm{f}}^{\alpha}(a) \overline{\mathrm{f}}_{\alpha}\left(\overline{\mathrm{f}}^{\beta}(b) \overline{\mathrm{f}}_{\beta}(c)\right)=a \star(b \star c) .
\end{aligned}
$$

We will see that $A_{\star}$ is a module algebra with respect to a deformed Hopf algebra $U g_{\star}$ or the isomorphic Hopf algebra $U g^{\mathcal{F}}$, (cf. text after (2.55), and Note 1 at the end of this section).

\section{Examples of twists}

Consider the element in $U g[[\lambda]] \otimes U g[[\lambda]]$ given by

$$
\mathcal{F}=e^{-\frac{i}{2} \lambda \theta^{\mu \nu} t_{\mu} \otimes t_{\nu}}
$$


where the elements $\left\{t_{\mu}\right\}$ generate an abelian subalgebra of $g$, and $\theta^{\mu \nu}$ is a constant matrix (usually antisymmetric and real in order to be compatible with conjugation). The inverse of $\mathcal{F}$ is

$$
\mathcal{F}^{-1}=e^{\frac{i}{2} \lambda \theta^{\mu \nu} t_{\mu} \otimes t_{\nu}}
$$

Then we have $(\Delta \otimes i d) \mathcal{F}=e^{-\frac{i}{2} \lambda \theta^{\mu \nu}\left(t_{\mu} \otimes 1 \otimes t_{\nu}+1 \otimes t_{\mu} \otimes t_{\nu}\right)}$ so that property (2.5) easily follows: $\mathcal{F}_{12}(\Delta \otimes i d) \mathcal{F}=e^{-\frac{i}{2} \lambda \theta^{\mu \nu}\left(t_{\mu} \otimes t_{\nu} \otimes 1+t_{\mu} \otimes 1 \otimes t_{\nu}+1 \otimes t_{\mu} \otimes t_{\nu}\right)}=\mathcal{F}_{23}(i d \otimes \Delta) \mathcal{F}$. Property (2.6) trivially holds.

Twists are not necessarily related to abelian Lie algebras. For example consider the elements $H, E, A, B$, that satisfy the Lie algebra relations

$$
\begin{aligned}
& {[H, E]=2 E, \quad[H, A]=\alpha A, \quad[H, B]=\beta B, \quad \alpha+\beta=2,} \\
& {[A, B]=E, \quad[E, A]=0, \quad[E, B]=0 .}
\end{aligned}
$$

Then the element

$$
\mathcal{F}=e^{\frac{1}{2} H \otimes \ln (1+\lambda E)} e^{\lambda A \otimes B \frac{1}{1+\lambda E}}
$$

is a twist and gives a well defined $\star$-product on the algebra of functions on $M$. These twists are known as extended Jordanian deformations [21]. Jordanian deformations [19, 20] are obtained setting $A=B=0$ (and keeping the relation $[H, E]=2 E$ ).

\section{$2.1 \star$-Noncommutative Manifolds}

Definition 1. A $\star$-noncommutative manifold is a quadruple $(M, g, \mathcal{F}, \rho)$ where $M$ is a smooth manifold, $g$ is a Lie algebra with twist $\mathcal{F} \in U g[[\lambda]] \otimes U g[[\lambda]]$ and $\rho: g \rightarrow \Xi$ is a homomorphism of $g$ in the Lie algebra $\Xi$ of vectorfields on $M$.

Usually we just consider the image $\rho(g)$ of $g$ in $\Xi$. Then the $\star$-noncommutative manifold is defined by the couple $(M, \mathcal{F})$ where $\mathcal{F} \in U \Xi[[\lambda]] \otimes U \Xi[[\lambda]]$.

We now use the twist to deform the commutative geometry on a manifold $M$ (vectorfields, 1-forms, exterior algebra, tensor algebra, symmetry algebras, covariant derivatives etc.) into the twisted noncommutative one. The guiding principle is the observation that every time we have a bilinear map

$$
\mu: X \times Y \rightarrow Z
$$

where $X, Y, Z$ are vectorspaces, and where there is an action of the Lie algebra $g$ (and therefore of $\mathcal{F}^{-1}$ ) on $X$ and $Y$ we can compose this map with the action of the twist. In this way we obtain a deformed version $\mu_{\star}$ of the initial bilinear map $\mu$ :

$$
\mu_{\star}:=\mu \circ \mathcal{F}^{-1},
$$




$$
\begin{aligned}
\mu_{\star}: X \times Y & \rightarrow Z \\
(\mathrm{x}, \mathrm{y}) & \mapsto \mu_{\star}(\mathrm{x}, \mathrm{y})=\mu\left(\overline{\mathrm{f}}^{\alpha}(\mathrm{x}), \overline{\mathrm{f}}_{\alpha}(\mathrm{y})\right)
\end{aligned}
$$

The action of $g$ on the vectorspaces $X, Y, Z$ we consider is given by representing $g$ as a Lie subalgebra of the Lie algebra $\Xi$ of vectorfieds on the manifold $M$. The action of vectorfields will always be via the Lie derivative.

Algebra of Functions $A_{\star}$. If $X=Y=Z=F u n(M)$ where $A \equiv F u n(M)$ is the space of functions on the manifold $M$, we obtain the star-product formula, for all $a, b \in A$

$$
a \star b=\mu \circ \mathcal{F}^{-1}(a \otimes b)=\overline{\mathrm{f}}^{\alpha}(a) \overline{\mathrm{f}}_{\alpha}(b) .
$$

We denote by $A_{\star}$ the noncommutative algebra of functions with the $\star$-product. We also introduce the universal $\mathcal{R}$-matrix

$$
\mathcal{R}:=\mathcal{F}_{21} \mathcal{F}^{-1}
$$

where by definition $\mathcal{F}_{21}=\mathrm{f}_{\alpha} \otimes \mathrm{f}^{\alpha}$. In the sequel we use the notation

$$
\mathcal{R}=R^{\alpha} \otimes R_{\alpha} \quad, \quad \mathcal{R}^{-1}=\bar{R}^{\alpha} \otimes \bar{R}_{\alpha} .
$$

The $\mathcal{R}$-matrix measures the noncommutativity of the $\star$-product. Indeed it is easy to see that

$$
h \star g=\bar{R}^{\alpha}(g) \star \bar{R}_{\alpha}(h) .
$$

The permutation group in noncommutative space is naturally represented by $\mathcal{R}$. Formula (2.24) says that the $\star$-product is $\mathcal{R}$-commutative in the sense that if we permute (exchange) two functions using the $\mathcal{R}$-matrix action then the result does not change.

Basic Example. If $M=\mathbb{R}^{n}$ a main example is given by considering the twist

$$
\mathcal{F}=e^{-\frac{i}{2} \lambda \theta^{\mu \nu} \frac{\partial}{\partial x^{\mu}} \otimes \frac{\partial}{\partial x^{\nu}}}
$$

The $\star$-product that the twist $\mathcal{F}$ induces on the algebra of functions on $\mathbb{R}^{n}$ is the Moyal-Weyl $\star$-product,

$$
(f \star g)(x)=\left.e^{\frac{i}{2} \lambda \theta^{\mu \nu} \frac{\partial}{\partial x^{\mu}} \frac{\partial}{\partial y^{\nu}}} f(x) g(y)\right|_{y=x} .
$$

Vectorfields $\Xi_{\star}$. We now deform the $A$-module of vectorifelds. According to (2.20) the product $\mu: A \otimes \Xi \rightarrow \Xi$ is deformed into the product

$$
h \star v=\overline{\mathrm{f}}^{\alpha}(h) \overline{\mathrm{f}}_{\alpha}(v) .
$$


Here $\overline{\mathrm{f}}^{\alpha} \in U g \subset U \Xi$. Its action on vectorfiels $\overline{\mathrm{f}}^{\alpha}(v)$ is given by the iterated use of the Lie derivative $t(v)=[t, v]$, (e.g. $\left.\left(t^{\prime} t\right)(v)=t^{\prime}(t(v))=\left[t^{\prime},[t, v]\right]\right)$. It is the adjoint action. The cocycle condition (2.5) implies that this new product is compatible with the $\star$-product in $A_{\star}$. We have thus constructed the $A_{\star}$ module of vectorfields. We denote it by $\Xi_{\star}$. As vectorspaces $\Xi=\Xi_{\star}$, but $\Xi$ is an $A$ module while $\Xi_{\star}$ is an $A_{\star}$ module.

1-forms $\Omega_{\star}$. The space of 1 -forms $\Omega$ becomes also an $A_{\star}$ module, with the product between functions and 1-forms given again by following the general prescription (2.20):

$$
h \star \omega:=\overline{\mathrm{f}}^{\alpha}(h) \overline{\mathrm{f}}_{\alpha}(\omega) .
$$

The action of $\overline{\mathrm{f}}_{\alpha}$ on forms is given by iterating the Lie derivative action of the vectorfield $t_{\mu}$ on forms. Functions can be multiplied from the left or from the right, if we deform the multiplication from the right we obtain the new product

$$
\omega \star h:=\overline{\mathrm{f}}^{\alpha}(\omega) \overline{\mathrm{f}}_{\alpha}(h)
$$

and we "move $h$ to the right" with the help of the $R$-matrix,

$$
\omega \star h=\bar{R}^{\alpha}(h) \star \bar{R}_{\alpha}(\omega) .
$$

We have defined the $A_{\star}$-bimodule of 1-forms.

Tensorfields $\mathcal{T}_{\star}$. Tensorfields form an algebra with the tensorproduct $\otimes$. We define $\mathcal{T}_{\star}$ to be the noncommutative algebra of tensorfields. As vectorspaces $\mathcal{T}=\mathcal{T}_{\star}$ the noncommutative tensorproduct is obtained by applying (2.20):

$$
\tau \otimes_{\star} \tau^{\prime}:=\overline{\mathrm{f}}^{\alpha}(\tau) \otimes \overline{\mathrm{f}}_{\alpha}\left(\tau^{\prime}\right) .
$$

Associativity of this product follows from the cocycle condition (2.5)).

Exterior forms $\Omega_{\star}^{*}=\oplus_{p} \Omega_{\star}^{p}$. Exterior forms form an algebra with product $\wedge: \Omega^{*} \times \Omega^{\cdot} \rightarrow$ $\Omega^{*}$. We $\star$-deform the wedge product into the $\star$-wedge product,

$$
\vartheta \wedge_{\star} \vartheta^{\prime}:=\overline{\mathrm{f}}^{\alpha}(\vartheta) \wedge \overline{\mathrm{f}}_{\alpha}\left(\vartheta^{\prime}\right) \text {. }
$$

We denote by $\Omega_{\star}^{*}$ the linear space of forms equipped with the wedge product $\wedge_{\star}$.

As in the commutative case exterior forms are totally $\star$-antisymmetric contravariant tensorfields. For example the 2 -form $\omega \wedge_{\star} \omega^{\prime}$ is the $\star$-antisymmetric combination

$$
\omega \wedge_{\star} \omega^{\prime}=\omega \otimes_{\star} \omega^{\prime}-\bar{R}^{\alpha}\left(\omega^{\prime}\right) \otimes_{\star} \bar{R}_{\alpha}(\omega) .
$$

Since Lie derivative and exterior derivative commute, the exterior derivative $\mathrm{d}: A \rightarrow \Omega$ satisfies the Leibniz rule $\mathrm{d}(h \star g)=\mathrm{d} h \star g+h \star \mathrm{d} g$, and is therefore also the $\star$-exterior derivative. The same argument shows that the de Rham cohomology ring is undeformed. 
$\star$-Pairing. We now consider the bilinear map $\langle\rangle:, \Xi \times \Omega \rightarrow A,(v, \omega) \mapsto\langle v, \omega\rangle$, where, using local coordinates, $\left\langle v^{\mu} \partial_{\mu}, \omega_{\nu} d x^{\nu}\right\rangle=v^{\mu} \omega_{\mu}$. Always according to the general prescription (2.20) we deform this pairing into

$$
\begin{aligned}
\langle,\rangle_{\star}: \Xi_{\star} \times \Omega_{\star} & \rightarrow A_{\star}, \\
(\xi, \omega) & \mapsto\langle\xi, \omega\rangle_{\star}:=\left\langle\overline{\mathrm{f}}^{\alpha}(\xi), \overline{\mathrm{f}}_{\alpha}(\omega)\right\rangle .
\end{aligned}
$$

It is easy to see that due to the cocycle condition for $\mathcal{F}$ the $\star$-pairing satisfies the $A_{\star}$ linearity properties

$$
\begin{gathered}
\langle h \star u, \omega \star k\rangle_{\star}=h \star\langle u, \omega\rangle_{\star} \star k, \\
\langle u, h \star \omega\rangle_{\star}=\bar{R}^{\alpha}(h) \star\left\langle\bar{R}_{\alpha}(u), \omega\right\rangle_{\star} .
\end{gathered}
$$

Using the pairing $\langle,\rangle_{\star}$ we associate to any 1-form $\omega$ the left $A_{\star}$-linear map $\langle, \omega\rangle_{\star}$. Also the converse holds: any left $A_{\star}$-linear map $\Phi: \Xi_{\star} \rightarrow A_{\star}$ is of the form $\langle, \omega\rangle_{\star}$ for some $\omega$ (explicitly $\omega=\Phi\left(\partial_{\mu}\right) d x^{\mu}$ ).

The pairing can be extended to covariant tensors and contravariant ones. We first define in the undeformed case the pairing

$$
\left\langle u^{\prime \prime} \ldots \otimes u^{\prime} \otimes u, \theta \ldots \otimes \theta^{\prime} \otimes \theta^{\prime \prime}\right\rangle=\langle u, \theta\rangle\left\langle u^{\prime}, \theta^{\prime}\right\rangle\left\langle u^{\prime \prime}, \theta^{\prime \prime}\right\rangle
$$

( $u, u^{\prime}, u^{\prime \prime}$ vectorfields, $\theta, \theta^{\prime}, \theta^{\prime \prime}$ 1-forms) that is obtained by first contracting the innermost elements. Using locality and linearity this pairing is extended to any covariant and contravariant $n$-tensors. It is this onion-like structure pairing that naturally generalizes to the noncommutative case.

The $\star$-pairing is defined by

$$
\langle\tau, \rho\rangle_{\star}:=\left\langle\overline{\mathrm{f}}^{\alpha}(\tau), \overline{\mathrm{f}}_{\alpha}(\rho)\right\rangle .
$$

Using the cocycle condition for the twist $\mathcal{F}$ and the onion like structure of the undeformed pairing we have the property

$$
\left\langle\tau \otimes_{\star} u, \theta \otimes_{\star} \rho\right\rangle_{\star}:=\left\langle\tau,\langle u, \theta\rangle_{\star} \star \rho\right\rangle_{\star} .
$$

This property uniquely characterizes the pairing because we also have the $A_{\star}$ linearity properties

$$
\begin{gathered}
\langle h \star \tau, \rho \star k\rangle_{\star}=h \star\langle\tau, \rho\rangle_{\star} \star k, \\
\langle\tau, h \star \rho\rangle_{\star}=\bar{R}^{\alpha}(h) \star\left\langle\bar{R}_{\alpha}(\tau), \rho\right\rangle_{\star} .
\end{gathered}
$$

$\star$-Hopf algebra of vectorfields $U \Xi_{\star}$. Consider the universal enveloping algebra $U \Xi$ of vectorfields on $M$ (infinitesimal diffeomorphisms), where $\Xi$ is the Lie algebra of vectorfields 
with the usual Lie bracket $[u, v](h)=u(v(h))-v(u(h))$. In order to construct the deformed algebra of vectorfields we apply the recepy (2.20) and deform the product in $U \Xi$ into the new product

$$
\xi \star \zeta=\overline{\mathrm{f}}^{\alpha}(\xi) \overline{\mathrm{f}}_{\alpha}(\zeta) .
$$

We call $U \Xi_{\star}$ the new algebra with product $\star$. As vectorspaces $U \Xi=U \Xi_{\star}$. Since any sum of products of vectorfields in $U \Xi$ can be rewritten as sum of $\star$-products of vectorfields via the formula $u v=\mathrm{f}^{\alpha}(u) \star \mathrm{f}_{\alpha}(v)$, vectorfields $u$ generate the algebra $U \Xi_{\star}$.

It turns out [16] that $U \Xi_{\star}$ has also a Hopf algebra structure. We describe it by giving the coproduct, the inverse of the antipode and the counit on the generators $u$ of $U \Xi_{\star}$ :

$$
\begin{gathered}
\Delta_{\star}(u)=u \otimes 1+\mathrm{X}_{\bar{R}^{\alpha}} \otimes \bar{R}_{\alpha}(u) \\
S_{\star}^{-1}(u)=-\bar{R}^{\alpha}(u) \star \mathrm{X}_{\bar{R}_{\alpha}} . \\
\varepsilon_{\star}(u)=\varepsilon(u)=0,
\end{gathered}
$$

where, for all $\xi \in U \Xi, \mathbf{X}_{\xi}=\overline{\mathrm{f}}^{\alpha} \xi \mathrm{f}^{\beta} S\left(\mathrm{f}_{\beta}\right) S^{-1}\left(\overline{\mathrm{f}}_{\alpha}\right)$. The map $\mathbf{X}: U \Xi \rightarrow U \Xi$ is invertible and it can be shown [22], that its inverse $X^{-1}$ is

$$
\mathrm{X}_{\xi}^{-1}=\overline{\mathrm{f}}^{\alpha}(\xi) \overline{\mathrm{f}}_{\alpha}=: D(\xi) .
$$

In principle one could directly check that (2.43)-(2.45) define a bona fide Hopf algebra. Another way [16] is to show that the Hopf algebra $U \Xi_{\star}$ is isomorphic to the Hopf algebra $U \Xi^{\mathcal{F}}$ studied by [7]. This latter has the same algebra structure as $U \Xi$. The new coproduct $\Delta^{\mathcal{F}}$, antipode $S^{\mathcal{F}}$ and the counit are given by

$$
\begin{gathered}
\Delta^{\mathcal{F}}(\xi)=\mathcal{F} \Delta(\xi) \mathcal{F}^{-1} . \\
S^{\mathcal{F}}(\xi)=\chi S(\xi) \chi^{-1} . \\
\varepsilon^{\mathcal{F}}(u)=\varepsilon(u)=0,
\end{gathered}
$$

where $\chi:=\mathrm{f}^{\alpha} S\left(\mathrm{f}_{\alpha}\right), \chi^{-1}=S\left(\overline{\mathrm{f}}^{\alpha}\right) \overline{\mathrm{f}}_{\alpha}$. The isomorphism is given by the map $D$ :

$$
\begin{aligned}
& D(\xi \star \zeta)=D(\xi) D(\zeta), \\
& \Delta_{\star}=\left(D^{-1} \otimes D^{-1}\right) \circ \Delta^{\mathcal{F}} \circ D, \\
& S_{\star}=D^{-1} \circ S^{\mathcal{F}} \circ D .
\end{aligned}
$$

Summarizing we have encountered the Hopf algebras

$$
(U \Xi, \cdot, \Delta, S, \varepsilon), \quad\left(U \Xi^{\mathcal{F}}, \cdot, \Delta^{\mathcal{F}}, S^{\mathcal{F}}, \varepsilon\right), \quad\left(U \Xi_{\star}, \star, \Delta_{\star}, S_{\star}, \varepsilon\right) .
$$


The first is cocommutative, the second is triangular and is obtained twisting the first, the third is triangular and isomorphic to the second. The remarkable fact about $U \Xi_{\star}$ is the Leibniz rule for vectorfields (2.43). We have that $\bar{R}_{\alpha}(u)$ is again a vectorfield so that

$$
\Delta_{\star}\left(\Xi_{\star}\right) \subset \Xi_{\star} \otimes 1+U \Xi_{\star} \otimes \Xi_{\star} .
$$

This is a fundamental property for the construction of a deformed differential calculus à la Woronowicz [23]. Note that the coproduct $\Delta^{\mathcal{F}}(u)$ does not have this property.

There is a natural action (Lie derivative) of $\Xi_{\star}$ on the space of functions $A_{\star}$. It is given once again by combining the usual Lie derivative on functions $\mathcal{L}_{u}(h)=u(h)$ with the twist $\mathcal{F}$ as in (2.20),

$$
\mathcal{L}_{u}^{\star}(h):=\overline{\mathrm{f}}^{\alpha}(u)\left(\overline{\mathrm{f}}_{\alpha}(h)\right) .
$$

The action $\mathcal{L}^{\star}$ of $\Xi_{\star}$ on $A_{\star}$ can be extended to all $U \Xi_{\star}$. The map $\mathcal{L}^{\star}$ is an action of $U \Xi_{\star}$ on $A_{\star}$, i.e. it represents the algebra $U \Xi_{\star}$ as differential operators on functions because

$$
\mathcal{L}_{u}^{\star}\left(\mathcal{L}_{v}^{\star}(h)\right)=\mathcal{L}_{u \star v}^{\star}(h) .
$$

We also have that $\mathcal{A}_{\star}$ is a $U \Xi_{\star}$ module algebra because $\mathcal{L}_{u}^{\star}$ is a deformed derivation of the algebra $\mathcal{A}_{\star}$ (cf. (2.14) ). Indeed in accordance with the coproduct formula (2.43) the differential operator $\mathcal{L}_{u}^{\star}$ satisfies the deformed Leibniz rule

$$
\mathcal{L}_{u}^{\star}(h \star g)=\mathcal{L}_{u}^{\star}(h) \star g+\bar{R}^{\alpha}(h) \star \mathcal{L}_{\bar{R}_{\alpha}(u)}^{\star}(g) .
$$

We conclude that $A_{\star}$ is a $U \Xi_{\star}$ module algebra.

This construction holds in general, see [16]: the deformed algebras of functions $A_{\star}$, of tensorfields $\mathcal{T}_{\star}$, of exterior forms $\Omega_{\star}^{*}$ and of vectorfields $U \Xi_{\star}$ are all $U \Xi_{\star}$ module algebras. The $U \Xi_{\star}$ action is always given by the $\star$-Lie derivative

$$
\mathcal{L}_{u}^{\star}:=\mathcal{L}_{\overline{\mathrm{f}}^{\alpha}(u)} \circ \overline{\mathrm{f}}^{\alpha} .
$$

The module property reads

$$
\mathcal{L}_{u}^{\star} \circ \mathcal{L}_{v}^{\star}=\mathcal{L}_{u \star v}^{\star}
$$

the compatibility with the algebra structure is the deformed Leibniz rule that correspond to the coproduct (2.43).

$\star$-Lie algebra of vectorfields $\Xi_{\star}$. In the case the deformation is given by a twist we have a natural candidate for the Lie algebra of the Hopf algebra $U \Xi_{\star}$. We apply the recepy (2.20) and deform the Lie algebra product [, ] into

$$
\begin{aligned}
{[]_{\star}: \quad \Xi \times \Xi } & \rightarrow \Xi \\
(u, v) & \mapsto[u, v]_{\star}:=\left[\overline{\mathrm{f}}^{\alpha}(u), \overline{\mathrm{f}}_{\alpha}(v)\right] .
\end{aligned}
$$


Notice that this $\star$-Lie bracket is just the $\star$-Lie derivative,

$$
[u, v]_{\star}:=\left[\overline{\mathrm{f}}^{\alpha}(u), \overline{\mathrm{f}}_{\alpha}(v)\right]=\mathcal{L}_{\overline{\mathrm{f}}^{\alpha}(u)}\left(\overline{\mathrm{f}}_{\alpha}(v)\right)=\mathcal{L}_{u}^{\star}(v) .
$$

In $U \Xi_{\star}$ it can be realized as a deformed commutator

$$
\begin{aligned}
{[u, v]_{\star} } & =\left[\overline{\mathrm{f}}^{\alpha}(u), \overline{\mathrm{f}}_{\alpha}(v)\right]=\overline{\mathrm{f}}^{\alpha}(u) \overline{\mathrm{f}}_{\alpha}(v)-\overline{\mathrm{f}}_{\alpha}(v) \overline{\mathrm{f}}^{\alpha}(u) \\
& =u \star v-\bar{R}^{\alpha}(v) \star \bar{R}_{\alpha}(u) .
\end{aligned}
$$

It is easy to see that the bracket $[,]_{\star}$ has the $\star$-antisymmetry property

$$
[u, v]_{\star}=-\left[\bar{R}^{\alpha}(v), \bar{R}_{\alpha}(u)\right]_{\star} .
$$

This can be shown as follows $[u, v]_{\star}=\left[\overline{\mathrm{f}}^{\alpha}(u), \overline{\mathrm{f}}_{\alpha}(v)\right]=-\left[\overline{\mathrm{f}}_{\alpha}(v), \overline{\mathrm{f}}^{\alpha}(u)\right]=-\left[\bar{R}^{\alpha}(v), \bar{R}_{\alpha}(u)\right]_{\star}$. A $\star$-Jacoby identity can be proven as well

$$
\left[u,[v, z]_{\star}\right]_{\star}=\left[[u, v]_{\star}, z\right]_{\star}+\left[\bar{R}^{\alpha}(v),\left[\bar{R}_{\alpha}(u), z\right]_{\star}\right]_{\star} .
$$

The appearence of the $R$-matrix $\mathcal{R}^{-1}=\bar{R}^{\alpha} \otimes \bar{R}_{\alpha}$ is not unexpected. We have seen that $\mathcal{R}^{-1}$ encodes the noncommutativity of the $\star$-product $h \star g=\bar{R}^{\alpha}(g) \star \bar{R}_{\alpha}(h)$ so that $h \star g$ do $\mathcal{R}^{-1}$-commute. Then it is natural to define $\star$-commutators using the $\mathcal{R}^{-1}$-matrix. In other words, the representation of the permutation group to be used on twisted noncommutative spaces is the one given by the $\mathcal{R}^{-1}$ matrix.

Furthermore it can be shown that the braket $[u, v]_{\star}$ is the $\star$-adjoint action of $u$ on $v$,

$$
[u, v]_{\star}=a d_{u}^{\star} v=u_{1_{\star}} \star v \star S\left(u_{2_{\star}}\right)
$$

here we have used the coproduct notation $\Delta_{\star}(u)=u_{1_{\star}} \otimes u_{2_{\star}}$. More in general the $\star$-adjoint action is the adjoint action in the Hopf algebra $U \Xi_{\star}$, it is given by, for all $\xi, \zeta \in U \Xi_{\star}$,

$$
a d_{\xi}^{\star} \zeta:=\xi_{1_{\star}} \star \zeta \star S_{\star}\left(\xi_{2_{\star}}\right)
$$

where we used the coproduct notation $\Delta_{\star}(\xi)=\xi_{1_{\star}} \otimes \xi_{2_{\star}}$.

We call $\left(\Xi,[,]_{\star}\right)$ the $\star$-Lie algebra of vectorfields because is a linear subspace of $U \Xi_{\star}$ such that

$$
\begin{aligned}
\text { i) } & \Xi_{\star} \text { generates } U \Xi_{\star}, \\
\text { ii) } & \Delta_{\star}\left(\Xi_{\star}\right) \subset \Xi_{\star} \otimes 1+U \Xi_{\star} \otimes \Xi_{\star}, \\
\text { iii) } & {\left[\Xi_{\star}, \Xi_{\star}\right]_{\star} \subset \Xi_{\star} . }
\end{aligned}
$$

Property $i$ i) implies a minimal deformation of the Leibniz rule. Property $i i i$ ) is the closure of $\Xi_{\star}$ under the adjoint action. These are the natural conditions that according to [23] a

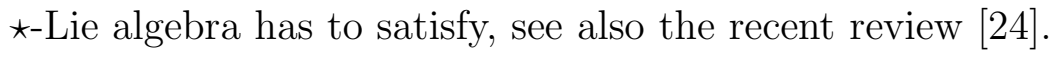


Note 1. The construction of the Hopf algebras $U \Xi_{\star}, U \Xi^{\mathcal{F}}$ and of the the quantum Lie algebra $\left(\Xi,[,]_{\star}\right)$ is based only on the twist $\mathcal{F} \in U \xi \otimes U \Xi$ (the Lie derivative is just the adjoint action). Given any Lie algebra $g$ and a twist $\mathcal{F} \in U g \otimes U g$ we similarly have the Hopf algebras $U g_{\star}, U g^{\mathcal{F}}$ and the quantum Lie algebra $\left(g,[,]_{\star}\right)$.

\section{Covariant Derivative, Torsion and Curvature}

The noncommutative differential geometry set up in the previous section allows to develop the formalism of covariant derivative, torsion and curvature just by following the usual classical formalism.

On functions the covariant derivative equals the Lie derivative. Requiring that this holds in the $\star$-noncommutative case as well we immediately know the action of the $\star$ covariant derivative on functions, and in particular the Leibniz rule it has to satisfy. More in general we define the $\star$-covariant derivative $\nabla_{u}^{\star}$ along the vector field $u \in \Xi$ to be the linear map $\nabla_{u}^{\star}: \Xi_{\star} \rightarrow \Xi_{\star}$ such that for all $u, v, z \in \Xi_{\star}, h \in A_{\star}$ :

$$
\begin{aligned}
& \nabla_{u+v}^{\star} z=\nabla_{u}^{\star} z+\nabla_{v}^{\star} z, \\
& \nabla_{h \star u}^{\star} v=h \star \nabla_{u}^{\star} v, \\
& \nabla_{u}^{\star}(h \star v)=\mathcal{L}_{u}^{\star}(h) \star v+\bar{R}^{\alpha}(h) \star \nabla_{\bar{R}_{\alpha}(u)} v
\end{aligned}
$$

This last expression is well defined because we have used the coproduct (2.43) that insures that $\bar{R}_{\alpha}(u)$ is again a vectorfield.

The covariant derivative is extended to tensorfields using the deformed Leibniz rule

$$
\nabla_{u}^{\star}\left(v \otimes_{\star} z\right)=\nabla_{u}^{\star}(v) \otimes_{\star} z+\bar{R}^{\alpha}(v) \otimes_{\star} \nabla_{\bar{R}_{\alpha}(u)}^{\star}(z) .
$$

The torsion $T$ and the curvature $\mathrm{R}$ associated to a connection $\nabla^{\star}$ are the linear maps $\mathrm{T}: \Xi_{\star} \times \Xi_{\star} \rightarrow \Xi_{\star}$, and $\mathrm{R}^{\star}: \Xi_{\star} \times \Xi_{\star} \times \Xi_{\star} \rightarrow \Xi_{\star}$ defined by

$$
\begin{aligned}
\mathrm{T}(u, v) & :=\nabla_{u}^{\star} v-\nabla_{\bar{R}^{\alpha}(v)}^{\star} \bar{R}_{\alpha}(u)-[u, v]_{\star}, \\
\mathrm{R}(u, v, z) & :=\nabla_{u}^{\star} \nabla_{v}^{\star} z-\nabla_{\bar{R}^{\alpha}(v)}^{\star} \nabla_{\bar{R}_{\alpha}(u)}^{\star} z-\nabla_{[u, v]_{\star}}^{\star},
\end{aligned}
$$

for all $u, v, z \in \Xi_{\star}$. From the $\star$-antisymmetry property of the bracket $[,]_{\star}$, see (2.62), it easily follows that the torsion $\mathrm{T}$ and the curvature $\mathrm{R}$ have the following $\star$-antisymmetry property

$$
\begin{aligned}
\mathrm{T}(u, v) & =-\mathrm{T}\left(\bar{R}^{\alpha}(v), \bar{R}_{\alpha}(u)\right), \\
\mathrm{R}(u, v, z) & =-\mathrm{R}\left(\bar{R}^{\alpha}(v), \bar{R}_{\alpha}(u), z\right) .
\end{aligned}
$$


The presence of the $R$-matrix in the definition of torsion and curvature insures that $\mathrm{T}$ and $\mathrm{R}$ are left $A_{\star}$-linear maps, i.e.

$$
\mathrm{T}(f \star u, v)=f \star \mathrm{T}(u, v) \quad, \quad \mathrm{T}(u, f \star v)=\bar{R}^{\alpha}(f) \star \mathrm{T}\left(\bar{R}_{\alpha}(u), v\right)
$$

and similarly for the curvature. The $A_{\star}$-linearity of $\mathrm{T}$ and $\mathrm{R}$ insures that we have a well defined torsion tensor and curvature tensor.

\section{Local coordinates description}

We denote by $\left\{e_{i}\right\}$ a local frame of vectorfields (subordinate to an open $U \subset M$ ) and by $\left\{\theta_{j}\right\}$ the dual frame of 1 -forms:

$$
\left\langle e_{i}, \theta^{j}\right\rangle_{\star}=\delta_{i}^{j}
$$

The coefficients $\mathrm{T}_{i j}{ }^{l}$ and $\mathrm{R}_{i j k}{ }^{l}$ of the torsion and curvature tensors with respect to this local frame are uniquely defined by the following expressions

$$
\begin{aligned}
& \mathrm{T}=\theta^{j} \otimes_{\star} \theta^{i} \star \mathrm{T}_{i j}{ }^{l} \otimes_{\star} e_{l}, \\
& \mathrm{R}=\theta^{k} \otimes_{\star} \theta^{j} \otimes_{\star} \theta^{i} \star \mathrm{R}_{i j k}{ }^{l} \otimes_{\star} e_{l},
\end{aligned}
$$

so that $\mathrm{T}_{i j}^{l}=\left\langle\mathrm{T}\left(e_{i}, e_{j}\right), \theta^{l}\right\rangle_{\star}, \mathrm{R}_{i j k}^{l}=\left\langle\mathrm{R}\left(e_{i}, e_{j}, e_{k}\right), \theta^{l}\right\rangle_{\star}$. We also have [25]

$$
\begin{aligned}
& \mathrm{T}=\frac{1}{2} \theta^{j} \wedge_{\star} \theta^{i} \star \mathrm{T}_{i j}^{l} \otimes_{\star} e_{l}, \\
& \mathrm{R}=\frac{1}{2} \theta^{k} \otimes_{\star} \theta^{j} \wedge_{\star} \theta^{i} \star \mathrm{R}_{i j k}{ }^{l} \otimes_{\star} e_{l} .
\end{aligned}
$$

We now define the connection coefficients $\Gamma_{i j}{ }^{k}$,

$$
\nabla_{e_{i}} e_{j}=\Gamma_{i j}^{k} \star e_{k}
$$

and define the connection forms $\omega_{i}^{j}$, the torsion forms $\Theta^{l}$ and the curvature forms $\Omega_{k}^{l}$ by

$$
\begin{aligned}
\omega_{i}^{j} & :=\theta^{k} \star \Gamma_{k i}^{j}, \\
\Theta^{l} & :=-\frac{1}{2} \theta^{j} \wedge_{\star} \theta^{i} \star \mathrm{T}_{i j}{ }^{l}, \\
\Omega_{k}^{l} & :=-\frac{1}{2} \theta^{j} \wedge_{\star} \theta^{i} \star \mathrm{R}_{k i j}^{l},
\end{aligned}
$$

It can be proven 25] that the Cartan structural equations hold

$$
\begin{aligned}
& \Theta^{l}=d \theta^{l}-\theta^{k} \wedge_{\star} \omega_{k}^{l}, \\
& \Omega_{k}^{l}=d \omega_{k}^{l}-\omega_{k}{ }^{m} \wedge_{\star} \omega_{m}^{l} .
\end{aligned}
$$

Differentiation of the Cartan structural equations gives the Bianchi identities

$$
\begin{aligned}
& d \Theta^{i}+\Theta^{j} \wedge_{\star} \omega_{j}{ }^{i}=\theta^{j} \wedge_{\star} \Omega_{j}{ }^{i}, \\
& d \Omega_{k}{ }^{l}+\Omega_{k}{ }^{m} \wedge_{\star} \omega_{m}{ }^{l}-\omega_{k}{ }^{m} \wedge_{\star} \Omega_{m}{ }^{l}=0 .
\end{aligned}
$$


The proof is as in the commutative case, for example $d \Theta^{i}=-\mathrm{d} \theta^{j} \wedge_{\star} \omega_{j}{ }^{i}+\theta^{j} \wedge_{\star} \mathrm{d} \omega_{j}{ }^{i}=$ $-\Theta^{j} \wedge_{\star} \omega_{j}{ }^{i}-\theta^{k} \wedge_{\star} \omega_{k}{ }^{j} \wedge_{\star} \omega_{j}{ }^{i}+\theta^{j} \wedge_{\star} \Omega_{j}{ }^{i}+\theta^{k} \wedge_{\star} \omega_{k}{ }^{j} \wedge_{\star} \omega_{j}{ }^{i}=-\Theta^{j} \wedge_{\star} \omega_{j}{ }^{i}+\theta^{j} \wedge_{\star} \Omega_{j}{ }^{i}$.

We conclude this section observing that along these lines one can also consider $\star$ Riemaniann geometry. In order to define a $\star$-metric we need to define $\star$-symmetric elements in $\Omega_{\star} \otimes_{\star} \Omega_{\star}$ where $\Omega_{\star}$ is the space of 1 -forms. Recalling that permutations are implemented with the $R$-matrix we see that $\star$-symmetric elements are of the form

$$
\omega \otimes_{\star} \omega^{\prime}+\bar{R}^{\alpha}\left(\omega^{\prime}\right) \otimes_{\star} \bar{R}_{\alpha}(\omega) .
$$

In particular any symmetric tensor in $\Omega \otimes \Omega$ is also a $\star$-symmetric tensor in $\Omega_{\star} \otimes_{\star} \Omega_{\star}$, indeed expansion of (3.84) gives $\overline{\mathrm{f}}^{\alpha}(\omega) \otimes \overline{\mathrm{f}}_{\alpha}\left(\omega^{\prime}\right)+\overline{\mathrm{f}}_{\alpha}\left(\omega^{\prime}\right) \otimes \overline{\mathrm{f}}^{\alpha}(\omega)$. Similarly for antisymmetric tensors.

As studied in [15, 16] it is possible to construct a torsionfree metric compatible connection and to consider the appropriate $\star$-contraction of the Riemann tensor that leads to a well defined Ricci tensor. One can therefore consider Einstein equations in vacuum, i.e. the vanshing of the Ricci tensor, where this last is seen as a function of the metric tensor.

\section{Deformed Poisson geometry}

In this section, developing the results of [18], we study $\star$-Poisson geometry. Let's first recall the very basic structures that we later deform.

\subsection{Poisson Bracket}

A Poisson structure on a manifold $M$ is a bilinear map

$$
\{,\}: \mathcal{A} \times \mathcal{A} \longrightarrow \mathcal{A}
$$

where $\mathcal{A}$ is the algebra of smooth functions on $M$. It satisfies

$$
\begin{array}{ll}
\{f, g\}=-\{g, f\} & \text { antisymmetry } \\
\{f,\{g, h\}\}+\{h,\{f, g\}\}+\{g,\{h, f\}\}=0 & \text { Jacobi identity } \\
\{f, g h\}=\{f, g\} h+g\{f, h\} & \text { Leibniz rule }
\end{array}
$$

The first two properties show that the Poisson bracket $\{$,$\} is a Lie bracket. We have$ the Lie algebra $(\mathcal{A},\{\}$,$) . We can therefore consider the universal enveloping algebra U \mathcal{A}$ that is a Hopf algebra. It is the algebra freely generated by the functions on $M$ modulo 
the ideal generated by the equivalence relation $f \cdot g-g \cdot f \sim\{f, g\}$. We denote by $\cdot$ the associative product in $U \mathcal{A}$, not to be confused with the product in $\mathcal{A}$.

The last property (Leibniz rule) shows that the map $\{f\}:, \mathcal{A} \rightarrow \mathcal{A}$ is a derivation of the algebra of functions $\mathcal{A}$. In other words we have an action of the Lie algebra $(\mathcal{A},\{\}$, on the algebra $\mathcal{A}$. As we recalled after (2.14) the action of $(\mathcal{A},\{\}$,$) on \mathcal{A}$ induces an action of $U \mathcal{A}$ on $\mathcal{A}$ so that $\mathcal{A}$ is a $U \mathcal{A}$-module algebra.

We have seen that a Poisson algebra can be equivalently defined as an associative algebra $\mathcal{A}$ that has also a compatible Lie bracket $\{$,$\} . The compatibility being that the$ associative algebra $\mathcal{A}$ is a module algebra with respect to the Hopf algebra $U \mathcal{A}$.

Since $\mathcal{A}$ is the algebra of smooth functions on $M$ we have the Lie algebra morphism

$$
\begin{aligned}
X:(\mathcal{A},\{,\}) & \rightarrow \Xi \\
f & \mapsto X_{f}:=\{f,\},
\end{aligned}
$$

$X_{f}$ is the Hamiltonian vectorfield associated to the "Hamiltonian" $f$. We have $\{f, g\}=$ $X_{f}(g)$. In this language the Jacoby identity reads

$$
X_{\{f, g\}}=\left[X_{f}, X_{g}\right]
$$

The morhism $X$ immediately lifts to the Hopf algebra morphism

$$
X: U \mathcal{A} \rightarrow U \Xi
$$

Concerning Hamiltonian vecorfields, from $\{f, g\}=X_{f}(g)=\left\langle X_{f}, \mathrm{~d} g\right\rangle$ and the antisymmetry property of the Poisson bracket we see that the vector field $X_{f}$ actually depends on $f$ only through its differential $\mathrm{d} f$, and we thus arrive at the Poisson bivector field $\Lambda$ that maps 1-forms into vectorfields according to

$$
\langle\Lambda, \mathrm{d} f\rangle=X_{f}
$$

We therefore have

$$
\langle\Lambda, \mathrm{d} f \otimes \mathrm{d} g\rangle=X_{f}(g)=\{f, g\} .
$$

Notice that we use the pairing $\langle u \otimes v, \mathrm{~d} f \otimes d g\rangle=\langle v, \mathrm{~d} f\rangle\langle u, \mathrm{~d} g\rangle$ ( $u$ and $v$ vectorfields) that is obtained by first contracting the innermost elements.

\section{$4.2 \star$-Poisson Bracket}

We now consider a noncommutative manifold $(M, g, \mathcal{F}, \rho)$ as defined in Section 3.1, and a Poisson structure on $M$. The natural compatibility condition between these two structures is to require the homomorphism $\rho: g \rightarrow \Xi$ to lift to a homomorphism $\tilde{\rho}: g \rightarrow(\mathcal{A},\{\}$,$) ,$ 
so that $X \circ \tilde{\rho}=\rho$. If this conditon holds then (we omit writing $\rho$ and $\tilde{\rho}$ maps) the twist $\mathcal{F} \in U \Xi \otimes U \Xi$ is the image under the map $X$ in (4.92) of a twist $\mathscr{F} \in U \mathcal{A} \otimes U \mathcal{A}$,

$$
\mathcal{F}=\mathrm{f}^{\alpha} \otimes \mathrm{f}_{\alpha}=X_{\mathrm{f}^{\alpha}} \otimes X_{\mathrm{f}_{\alpha}}, \quad \mathscr{F}=\mathrm{f}^{\alpha} \otimes \mathrm{f}_{\alpha} \in U \mathcal{A} \otimes U \mathcal{A} .
$$

We can then twist the universal enveloping algebra $U \mathcal{A}$ of the Lie algebra $(A,\{\}$,$) . We$ therefore obtain the Hopf algebra $U \mathcal{A}_{\star}$. The coproduct, the inverse of the antipode and the counit on functions are given by formulae (2.43)-(2.45) where the generic vectorfield $u$ is replaced by the generic function $h$ and the twist is given by $\mathscr{F}$. Of course we can also consider the twisted Hopf algebra $U \mathcal{A}^{\mathscr{F}}$; it is defined as in (2.47)-(2.49).

The $\star$-Poisson bracket is then defined as (cf. (2.59)),

$$
\{f, g\}_{\star}:=\left\{\overline{\mathrm{f}}^{\alpha}(f), \overline{\mathrm{f}}_{\alpha}(g)\right\}=\left\{\overline{\mathrm{f}}^{\alpha}(f), \overline{\mathrm{f}}_{\alpha}(g)\right\}
$$

The second equality is simply due to the fact that the action of $\mathscr{F}$ on functions is given by its image $\mathcal{F} \in U \Xi \otimes U \Xi$.

In full analogy with the construction of the quantum Lie algebra of vectorfields $\left(\Xi,[,]_{\star}\right)$, we have the quantum Lie algebra of classical obsevables $\left(\mathcal{A},\{,\}_{\star}\right)$. In particular the $\star-$ Poisson bracket is $\star$-antisymmetric and it satisfies the $\star$-Jacobi identity and the $\star$-Leibniz rulet:

$$
\begin{aligned}
\{f, g\}_{\star} & =-\left\{\overline{\mathrm{R}}^{\alpha}(g), \overline{\mathrm{R}}_{\alpha}(f)\right\}_{\star}, \\
\left\{f,\{g, h\}_{\star}\right\}_{\star} & =\left\{\{f, g\}_{\star}, h\right\}_{\star}+\left\{\overline{\mathrm{R}}^{\alpha}(g),\left\{\overline{\mathrm{R}}_{\alpha}(f), h\right\}_{\star}\right\}_{\star}, \\
\{f, g \star h\}_{\star} & =\{f, g\}_{\star} \star h+\overline{\mathrm{R}}^{\alpha}(g) \star\left\{\overline{\mathrm{R}}_{\alpha}(f), h\right\}_{\star} .
\end{aligned}
$$

From the $\star$-Leibniz rule (4.99) we see that $\{f$,$\} is a \star$-vectorfield, it is indeed $\mathcal{L}_{X_{f}}^{\star}$ :

$$
\mathcal{L}_{X_{f}}^{\star}(g)=\mathcal{L}_{\overline{\mathrm{f}}^{\alpha}\left(X_{f}\right)}\left(\overline{\mathrm{f}}_{\alpha}(g)\right)=\mathcal{L}_{X_{\overline{\mathrm{f}}^{\alpha}(f)}}\left(\overline{\mathrm{f}}_{\alpha}(g)\right)=\left\{\overline{\mathrm{f}}^{\alpha}(f), \overline{\mathrm{f}}_{\alpha}(g)\right\}=\{f, g\}_{\star} .
$$

In the second equality we used

$$
\overline{\mathrm{f}}^{\alpha}\left(X_{h}\right) \otimes \overline{\mathrm{f}}_{\alpha}=\overline{\mathrm{f}}^{\alpha}(\langle\Lambda, d f\rangle) \otimes \overline{\mathrm{f}}_{\alpha}=\left\langle\Lambda, d \overline{\mathrm{f}}^{\alpha}(f)\right\rangle \otimes \overline{\mathrm{f}}_{\alpha}=X_{\overline{\mathrm{f}}^{\alpha}(h)} \otimes \overline{\mathrm{f}}_{\alpha} ;
$$

this property holds because $\overline{\mathrm{f}}^{\alpha}$ is a sum of products of Hamiltonian vectorfields. We similarly have $\overline{\mathrm{f}}^{\alpha}\left(X_{h}\right) \otimes \overline{\mathrm{f}}_{\alpha}=X_{\overline{\mathrm{f}}^{\alpha}(h)} \otimes \overline{\mathrm{f}}_{\alpha}$. It follows that $X$ is a morphism between the quantum Lie algebras $\left(\mathcal{A},\{,\}_{\star}\right)$ and $\Xi_{\star}$ :

$$
\left[X_{f}, X_{g}\right]_{\star}=\left[\overline{\mathrm{f}}^{\alpha}\left(X_{f}\right), \overline{\mathrm{f}}_{\alpha}\left(X_{g}\right)\right]=\left[X_{\overline{\mathrm{f}}^{\alpha}(f)}, X_{\overline{\mathrm{f}}_{\alpha}(g)}\right]=X_{\left\{\overline{\mathrm{f}}^{\alpha}(f), \overline{\mathrm{f}}_{\alpha}(g)\right\}}=X_{\{f, g\}_{\star}} .
$$

\footnotetext{
${ }^{\dagger}$ In [18] we do not require the twist $\mathcal{F}$ to be the image of a twist $\mathscr{F} \in U \mathcal{A} \otimes U \mathcal{A}$. In general we therefore do not have a Hopf algebra $U \mathcal{A}_{\star}$. However we still have (4.97)-(4.99) because we impose the milder compatibility condition between the twist $\mathcal{F}$ and the Poisson tensor $\Lambda, \overline{\mathrm{f}}^{\alpha} \otimes \overline{\mathrm{f}}_{\alpha}(\Lambda)=1 \otimes \Lambda, \overline{\mathrm{f}}^{\alpha}(\Lambda) \otimes \overline{\mathrm{f}}_{\alpha}=$ $\Lambda \otimes 1$.
} 
Because of property (4.102) and of the coproduct rule (2.43) we have that for a twist $\mathcal{F}$ on $M$ with a compatible Poisson bracket Hamiltonian vector fields are a $\star$-Lie subalgebra of the $\star$-Lie algebra of vectorfields.

Similar techniques show that $X: U \mathcal{A}_{\star} \rightarrow U \Xi_{\star}$ is a homomorphism of Hopf algebras.

We can also consider the set of Hamiltonian vectorfields $\left\{X_{Q}\right\}$ that leave invariant a given Hamiltonian function $H$,

$$
\mathcal{L}_{X_{Q}}^{\star} H=0 \text {, i.e., }\{Q, H\}_{\star}=0 .
$$

In particular, recalling (4.102), we have $\left[X_{Q}, X_{H}\right]_{\star}=0$. If the Hamiltonian $H$ is invariant under the action of the twist, i.e., if, $\overline{\mathrm{f}}^{\alpha} \otimes \overline{\mathrm{f}}_{\alpha}(H)=1 \otimes H, \overline{\mathrm{f}}^{\alpha}(H) \otimes \overline{\mathrm{f}}_{\alpha}=H \otimes 1$ we have that $\{Q, H\}_{\star}=\{Q, H\}=\{H, Q\}_{\star}=0$ and $Q$ is a constant of motion. Using the $\star$-Jacoby identity we have that the $\star$-bracket $\left\{Q, Q^{\prime}\right\}_{\star}$ of two constants of motion is again a constant of motion. We conclude that the subspace of Hamiltonian vector fields $\left\{X_{Q}\right\}$ that leave invariant the Hamiltonian $H$ forms a $\star$-Lie subalgebra of the $\star$-Lie algebra of Hamiltonian vectorfields: the $\star$-symmetry algebra of constants of motion. The term constant of motion is appropriate because the natural noncommutative definition of time evolution

$$
\dot{f}=-\mathcal{L}_{X_{H}}^{\star} f=-\{H, f\}_{\star}
$$

reduces to the usual one $\dot{f}=-\{H, f\}$ for Hamiltonians $H$ invariant under the action of the twist.

\section{Example}

Let us consider the canonical bracket on phase space $M=\mathrm{T}^{*} \mathbb{R}^{n}$ with the usual coordinates $x^{1}, \ldots x^{n}, p_{1}, \ldots p_{n}$, (sum over $\ell=1, \ldots n$ is assumed)

$$
\{f, g\}:=\frac{\partial f}{\partial x^{\ell}} \frac{\partial g}{\partial p_{\ell}}-\frac{\partial f}{\partial p_{\ell}} \frac{\partial g}{\partial x^{\ell}} .
$$

Because of the onion like structure of the pairing and since $\left\langle\frac{\partial}{\partial x^{i}} \otimes \frac{\partial}{\partial p_{i}}, \mathrm{~d} f\right\rangle=\frac{\partial f}{\partial p_{i}} \frac{\partial}{\partial x^{i}}$, we have that the Poisson bivector field is

$$
\Lambda=\frac{\partial}{\partial p_{i}} \wedge \frac{\partial}{\partial x^{i}}=\frac{\partial}{\partial p_{i}} \otimes \frac{\partial}{\partial x^{i}}-\frac{\partial}{\partial x^{i}} \otimes \frac{\partial}{\partial p_{i}} .
$$

Let $\mathcal{A}=C^{\infty}(M)$ be the space of smooth complex valued functions on $M$. Consider the Lie algebra $(\mathcal{A},\{\}$,$) , its associated universal enveloping algebra (U \mathcal{A}, \cdot)$ where $\cdot$ is the product in $U \mathcal{A}$ (not to be confused with the product in $\mathcal{A}=C^{\infty}(M)$ ), and the twist (we absorb $\lambda$ in $\theta^{\ell s}$ )

$$
\mathscr{F}=\mathrm{e}^{-\frac{\mathrm{i}}{2} \theta_{s} p_{\ell} \otimes p_{s}} \in U \mathcal{A} \otimes U \mathcal{A}, \quad \ell, s=1, \ldots n
$$


The Hopf algebra map $X: U \mathcal{A} \rightarrow U \Xi$ that in particular maps elements $f$ of the Lie algebra $(\mathcal{A},\{\}$,$) , (i.e. functions), to the corrisponding Hamiltonian vectorfields X_{f}$, maps the twist $\mathscr{F}$ into the twist

$$
\mathcal{F}=\mathrm{e}^{-\frac{i}{2} \theta^{\ell s} \frac{\partial}{\partial x^{2}} \otimes \frac{\partial}{\partial x^{s}}} \in U \Xi \otimes U \Xi
$$

A simple calculation shows that the twisted Poisson bracket can be expressed as:

$$
\{f, g\}_{\star}=\frac{\partial f}{\partial x^{\ell}} \star \frac{\partial g}{\partial p_{\ell}}-\frac{\partial f}{\partial p_{\ell}} \star \frac{\partial g}{\partial x^{\ell}} .
$$

Any translation invariant Hamiltonian is compatible with the twist and the associated constants of motion form a $\star$-Lie algebra of constants of motions.

We see that this formalism is quite well suited for field theory Hamiltonians that have potentials like $\int \mathrm{d}^{3} x \bar{\Phi}(x) \star \Phi(x) \star \bar{\Phi}(x) \star \Phi(x)$ and are translation invariant under twists in phase space originating from twists on spacetime (actually on space) like $\mathcal{F}=$ $\mathrm{e}^{-\frac{i}{2} \theta^{i j} \frac{\partial}{\partial x^{i}} \otimes \frac{\partial}{\partial x^{j}}}$. This is the topic of the next section.

\section{$5 \quad$ Field Theory on noncommutative space}

We here generalize the twist setting to the case of an infinite number of degrees of freedom and apply this formalism to study a scalar field theory on noncommutative spacetime. We choose the easiest example, one scalar field with spacetime equal to $\mathbb{R}^{d+1}$ (Klein-Gordon field, or $\Phi^{4}$ theory). The relevant kinematical features of classical field theories and their $\hbar$-quantization are already present in this simple example.

\subsection{Classical Field Theory on noncommutative space}

The infinite dimensional phase space is described by the fields $\Phi(x)$ and $\Pi(x)$ with $x \in \mathbb{R}^{d}$ $\left(\mathbb{R}^{d+1}\right.$ being spacetime). The algebra $\mathrm{A}$ is an algebra of functionals, it is the algebra of functions on $N$ where in turn $N$ is the function space:

$$
N=\operatorname{Maps}\left(\mathbb{R}^{d} \rightarrow \mathbb{R}^{2}\right) .
$$

We define the Poisson bracket between the functionals $F, G \in \mathrm{A}$ to be

$$
\{F, G\}=\int \mathrm{d}^{d} x \frac{\delta F}{\delta \Phi} \frac{\delta G}{\delta \Pi}-\frac{\delta F}{\delta \Pi} \frac{\delta G}{\delta \Phi}
$$

The fields $\Phi(x)$ and $\Pi(x)$ for fixed $x$ can be considered themselves a family of functionals parametrized by $x \in \mathbb{R}^{n}$, for fixed $x, \Phi(x)$ is the functional (evaluation map) that 
associates to $\Phi$ and $\Pi$ the value $\Phi(x)$; similarly with $\Pi(x)$. Their brackets ar $\ddagger$

$$
\{\Phi(x), \Phi(y)\}=0, \quad\{\Pi(x), \Pi(y)\}=0, \quad\{\Phi(x), \Pi(y)\}=\delta(x-y) .
$$

Now let space $\mathbb{R}^{d}$ become the noncommutative Moyal space. The algebra of functions on $\mathbb{R}^{d}$ becomes noncommutative with noncommutativity given by the twist $\mathcal{F}=$ $\mathrm{e}^{-\frac{\mathrm{i}}{2} \theta^{i j} \frac{\partial}{\partial x^{i}} \otimes \frac{\partial}{\partial x^{j}}}$ (the Lie algebra $g$ in $(M, g, \mathcal{F}, \rho)$ in this case is that of translations).

The twist lifts to the algebra A of functionals [36] so that this latter too becomes noncommutative. This is achieved by lifting to $\mathrm{A}$ the action of infinitesimal translations. Explicitly $\frac{\partial}{\partial x^{i}}$ is lifted to $\partial_{i}^{*}$ acting on $\mathrm{A}$ as,

$$
\partial_{i}^{*} G:=-\int \mathrm{d}^{d} x \partial_{i} \Phi(x) \frac{\delta G}{\delta \Phi(x)}+\partial_{i} \Pi(x) \frac{\delta G}{\delta \Pi(x)} .
$$

Therefore on functionals the twist is represented as

$$
\mathcal{F}=\mathrm{e}^{-\frac{\mathrm{i}}{2} \theta^{i j} \int \mathrm{d}^{d} x\left(\partial_{i} \Phi \frac{\delta}{\delta \Phi(x)}+\partial_{i} \Pi \frac{\delta}{\delta \Pi(x)}\right) \otimes \int \mathrm{d}^{d} y\left(\partial_{j} \Phi \frac{\delta}{\delta \Phi(y)}+\partial_{j} \Pi \frac{\delta}{\delta \Pi(y)}\right)} .
$$

The associated $\star$-product is

$$
F \star G=\overline{\mathrm{f}}^{\alpha}(F) \overline{\mathrm{f}}_{\alpha}(G) .
$$

If $x=y$ the $\star$-product between the functionals $\Phi(x) \star \Phi(y)=(\Phi \star \Phi)(x)$ where this latter $\star$-product is the usual one for the function $\Phi$.

Like in the finite dimensional case, the $\star$-algebra of functionals $A_{\star}$ is a $U \Xi_{\star}$ module algebra where now $\Xi$ is the Lie algebra of vectorfields on $N$ (infinitesimal functional variations).

The vectorfield on functional space $\partial_{i}^{\star}$ is a Hamiltonian vectorfield with Hamiltonian functional $P_{i} \in$ A given by

$$
P_{i}=-\int \mathrm{d}^{d} y \Pi(y) \partial_{i} \Phi(y)
$$

Indeed $X_{P_{i}}=\int \mathrm{d}^{d} x \frac{\delta P_{i}}{\delta \Pi(x)} \frac{\delta}{\delta \Phi(x)}-\frac{\delta P_{i}}{\delta \Phi(x)} \frac{\delta}{\delta \Pi(x)}=\partial_{i}^{\star}$. The functional $P_{i}$ is just the momentum of the Klein-Gordon field as obtained via Noether theorem. The momenta $P_{i}$ are mutually commuting and the twist (5.5) is therefore the image via $X \otimes X: U \mathcal{A} \otimes U \mathcal{A} \rightarrow U \Xi \otimes U \Xi$ of the twist $\mathscr{F}=\mathrm{e}^{-\frac{\mathrm{i}}{2} \theta^{i j} P_{i} \otimes P_{j}} \in U \mathrm{~A} \otimes U \mathrm{~A}$. We therefore twist the Hopf algebra $U \mathrm{~A}$ in the Hopf algebra $U A_{\star}$. The corresponding quantum Lie algebra $\left(A,\{,\}_{\star}\right)$ is given by the deformed Poisson bracket, $\{,\}_{\star}: \mathrm{A} \otimes \mathrm{A} \rightarrow \mathrm{A}$,

$$
\{F, G\}_{\star}:=\left\{\overline{\mathrm{f}}^{\alpha}(F), \overline{\mathrm{f}}_{\alpha}(G)\right\} .
$$

\footnotetext{
${ }^{\ddagger}$ In order to avoid considering distributions we should work with smeared fields $\Phi(f)=\int \mathrm{d}^{d} x f(x) \Phi(x)$ and $\Pi(g)=\int \mathrm{d}^{d} x g(x) \Pi(x)$. The smeared version of the Poisson bracket is then $\{\Phi(f), \Pi(g)\}=$ $\int \mathrm{d}^{d} x f(x) g(x)$.
} 
This bracket satisfies the $\star$-antisymmetry, $\star$-Leibniz rule and $\star$-Jacoby identity

$$
\begin{aligned}
\{F, G\}_{\star} & =-\left\{\overline{\mathrm{R}}^{\alpha}(G), \overline{\mathrm{R}}_{\alpha}(F)\right\}_{\star} \\
\{F, G \star H\}_{\star} & =\{F, G\}_{\star} \star H+\overline{\mathrm{R}}^{\alpha}(G) \star\left\{\overline{\mathrm{R}}_{\alpha}(F), H\right\}_{\star} \\
\left\{F,\{G, H\}_{\star}\right\}_{\star} & =\left\{\{F, G\}_{\star}, H\right\}_{\star}+\left\{\overline{\mathrm{R}}^{\alpha}(G),\left\{\overline{\mathrm{R}}_{\alpha}(F), H\right\}_{\star}\right\}_{\star}
\end{aligned}
$$

In particular the $\star$-brackets among the fields are undeformed

$$
\begin{aligned}
& \{\Phi(x), \Pi(y)\}_{\star}=\{\Phi(x), \Pi(y)\}=\delta(x-y), \\
& \{\Phi(x), \Phi(y)\}_{\star}=\{\Phi(x), \Phi(y)\}=0, \\
& \{\Pi(x), \Pi(y)\}_{\star}=\{\Pi(x), \Pi(y)\}=0 .
\end{aligned}
$$

We prove the first relation

$$
\begin{aligned}
\{\Phi(x), \Pi(y)\}_{\star} & =\left\{\overline{\mathrm{f}}^{\alpha}(\Phi(x)), \overline{\mathrm{f}}_{\alpha}(\Pi(y))\right\} \\
& =\{\Phi(x), \Pi(y)\}-\frac{\mathrm{i}}{2} \theta^{i j}\left\{\int \mathrm{d}^{d} z \partial_{i} \Phi(z) \delta(x-z), \int \mathrm{d}^{d} w \partial_{j} \Pi(w) \delta(y-w)\right\}+O\left(\theta^{2}\right) \\
& =\{\Phi(x), \Pi(y)\}-\frac{\mathrm{i}}{2} \theta^{i j} \partial_{y^{j}} \partial_{x^{i}} \delta(x-y)+O\left(\theta^{2}\right) \\
& =\{\Phi(x), \Pi(y)\}
\end{aligned}
$$

the second term in the third line vanishes because of symmetry, as well as higher terms in $\theta^{i j}$.

We conclude that for Moyal-Weyl deformations the $\star$-Poisson bracket just among coordinates is unchanged. It is however important to stress that this is not the case in general. For nontrivial functionals of the fields we have

$$
\{F, G\}_{\star} \neq\{F, G\} \text {. }
$$

If we expand $\Phi$ and $\Pi$ in Fourier modes $\$$

$$
\begin{aligned}
\Phi(x) & =\int \frac{\mathrm{d}^{d} k}{(2 \pi)^{d} \sqrt{2 E_{k}}}\left(a(k) \mathrm{e}^{\mathrm{i} k x}+a^{*}(k) \mathrm{e}^{-\mathrm{i} k x}\right) \\
\Pi(x) & =\int \frac{\mathrm{d}^{d} k}{(2 \pi)^{d}}(-\mathrm{i} \hbar) \sqrt{\frac{E_{k}}{2}}\left(a(k) \mathrm{e}^{\mathrm{i} k x}-a^{*}(k) \mathrm{e}^{-\mathrm{i} k x}\right)
\end{aligned}
$$

where $E_{k}=\sqrt{m^{2}+\vec{p}^{2}}=\sqrt{m^{2}+\hbar^{2} \vec{k}^{2}}$, and $k x=\vec{k} \cdot \vec{x}=\sum_{i=1}^{d} k^{i} x^{i}$. We obtain the $\star$ commutation relations between the $a(k)$ functionals

$$
\begin{array}{rlrl}
a(k) \star a\left(k^{\prime}\right) & =\mathrm{e}^{-\frac{\mathrm{i}}{2} \theta^{i j} k_{i} k_{j}^{\prime}} a(k) a\left(k^{\prime}\right) & & a^{*}(k) \star a^{*}\left(k^{\prime}\right)=\mathrm{e}^{-\frac{\mathrm{i}}{2} \theta^{i j} k_{i} k_{j}^{\prime}} a^{*}(k) a^{*}\left(k^{\prime}\right), \\
a^{*}(k) \star a\left(k^{\prime}\right)=\mathrm{e}^{\frac{\mathrm{i}}{2} \theta^{i j} k_{i} k_{j}^{\prime}} a^{*}(k) a\left(k^{\prime}\right) & , & a(k) \star a^{*}\left(k^{\prime}\right)=\mathrm{e}^{\frac{\mathrm{i}}{2} \theta^{i j} k_{i} k_{j}^{\prime}} a(k) a^{*}\left(k^{\prime}\right) .
\end{array}
$$

\footnotetext{
${ }^{\S}$ We use the usual undeformed Fourier decomposition because indeed are the usual exponentials that, once we also add the time dependence part, solve the free field equation of motion on noncommutative space $\left(\hbar^{2} \partial^{\mu} \partial_{\mu}+m^{2}\right) \Phi=0$. This equation is the same as the one on commutative space because the $\star$-product enters only the interaction terms.
} 
We finally calculate the Poisson bracket among the Fourier modes using the definition (5.8) and the functional expressions of $a(k), a^{*}(k)$ in terms of $\Phi$ and $\Pi$. We obtain [18]

$$
\left\{a(k), a^{*}\left(k^{\prime}\right)\right\}_{\star}=-\frac{i}{\hbar}(2 \pi)^{d} \delta\left(k-k^{\prime}\right), \quad\left\{a(k), a\left(k^{\prime}\right)\right\}_{\star}=0, \quad\left\{a^{*}(k), a^{*}\left(k^{\prime}\right)\right\}_{\star}=0 .
$$

Although the twisted Poisson bracket is equal to the untwisted one for linear combinations of the Fourier modes, it yields a different result, involving nontrivial fases, as soon as we consider Poisson brackets of powers of $a, a^{*}$.

\subsection{Quantum Field theory on noncommutative space}

We now formulate the canonical quantization of scalar fields on noncommutative space. Associated to the algebra $\mathrm{A}$ of functionals $G[\Phi, \Pi]$ there is the algebra $\widehat{A}$ of functionals

$\hat{G}[\hat{\Phi}, \hat{\Pi}]$ on operator valued fields. We lift the twist to $\widehat{A}$ and then deform this algebra to $\widehat{\mathrm{A}}_{\star}$ by implementing once more the twist deformation principle (2.20). We denote by $\hat{\partial}_{i}$ the lift to $\widehat{A}$ of $\frac{\partial}{\partial x^{i}}$; for all $\hat{G} \in \widehat{A}$,

$$
\hat{\partial}_{i} \hat{G}:=-\int \mathrm{d}^{d} x \partial_{i} \hat{\Phi}(x) \frac{\delta \hat{G}}{\delta \hat{\Phi}(x)}+\partial_{i} \hat{\Pi}(x) \frac{\delta \hat{G}}{\delta \hat{\Pi}(x)}
$$

here $\partial_{i} \hat{\Phi}(x) \frac{\delta \hat{G}}{\delta \hat{\Phi}(x)}$ stands for $\int \mathrm{d}^{d} \ell \partial_{i} \Phi_{\ell}(x) \frac{\delta \hat{G}}{\delta \Phi_{\ell}(x)}$, where like in (5.17) we have expanded the operator $\hat{\Phi}(x)$ as $\int \mathrm{d}^{d} \ell \Phi_{\ell}(x) \hat{\mathrm{a}}(\ell)$ (and similarly for $\left.\hat{\Pi}(x)\right)$.

Consequently the twist on operator valued functionals reads $\hat{\mathcal{F}}=\mathrm{e}^{-\frac{i}{2} \theta^{i j} \hat{\partial}_{i} \otimes \hat{\partial}_{j}}$. The twist can further be lifted to the quantum algebra of observables. The momentum operators are

$$
\hat{P}_{i}=-\int \mathrm{d}^{d} y: \hat{\Pi}(y) \partial_{i} \hat{\Phi}(y):
$$

where the columns : : stem for normal ordering. The twist reads $\widehat{\mathscr{F}}=\mathrm{e}^{-\frac{\mathrm{i}}{2} \theta^{i j} \hat{P}_{i} \otimes \hat{P}_{j}}$. A similar twist has been independently considered in [31]. With this twist $\widehat{\mathscr{F}}$ we twist the Hopf algebra $U \widehat{\mathrm{A}}$ in the Hopf algebra $U \widehat{\mathrm{A}}_{\star}$. The corresponding quantum Lie algebra $\left(\widehat{A},[,]_{\star}\right)$ is given by the bracket, $[,]_{\star}: A \otimes A \rightarrow A$,

$$
[\hat{F}, \hat{G}]_{\star}:=\left[\overline{\mathrm{f}}^{\alpha}(\hat{F}), \overline{\mathrm{f}}_{\alpha}(\hat{G})\right]
$$

This deformed bracket satisfies the $\star$-antisymmetry, $\star$-Leibniz rule and $\star$-Jacoby identity (5.9), (5.10), (5.11) where all arguments are now operators and the bracket $\{,\}_{\star}$ is replaced with $[,]_{\star}$. This latter is the $\star$-commutator. Indeed recalling the definition of the $\mathcal{R}$-matrix it can be easily verified that

$$
[\hat{F}, \hat{G}]_{\star}=\hat{F} \star \hat{G}-\bar{R}^{\alpha}(\hat{G}) \star \bar{R}_{\alpha}(\hat{F})
$$


which is indeed the $\star$-commutator in $\hat{A}_{\star}$. This $\star$-commutator has been considered in [32].

We studied four Hopf algebras $U \mathrm{~A}, U \mathrm{~A}_{\star}, U \widehat{\mathrm{A}}, U \widehat{\mathrm{A}}_{\star}$, and their corresponding Lie alge$\operatorname{bras}(\mathrm{A},\{\}),,(\widehat{\mathrm{A}},[]),,\left(\mathrm{A}_{\star},\{,\}_{\star}\right),\left(\widehat{\mathrm{A}}_{\star},[,]_{\star}\right)$. Canonical quantization on noncommutative space is the map $\hbar_{\star}$ in the diagram

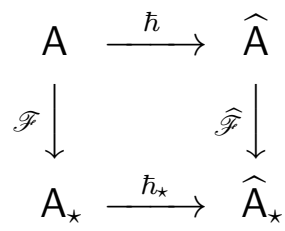

We define canonical quantization on nocommutative space by requiring this diagram to be commutative as a diagram among the vectorspaces $A, \widehat{A}, A_{\star}, \widehat{A}_{\star}$. Notice that the vertical maps, that with abuse of notation we have called $\mathscr{F}$ and $\widehat{\mathscr{F}}$, are the identity map, indeed $\mathrm{A}=\mathrm{A}_{\star}$ and $\widehat{\mathrm{A}}=\widehat{\mathrm{A}}_{\star}$ as vectorspaces. Therefore we have $\hbar_{\star}=\hbar$. The map $\hbar_{\star}$ satisfies a $\star$-correspondence principle because $\star$-Poisson brackets go into $\star$-commutators at leading order in $\hbar$

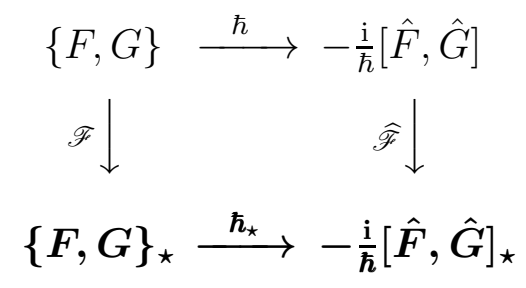

Indeed recall the definitions of the $\star$-Poisson bracket and of the $\star$-commutator and compute

$$
\{F, G\}_{\star}=\left\{\overline{\mathrm{f}}^{\alpha}(F), \overline{\mathrm{f}}_{\alpha}(G)\right\} \stackrel{\hbar}{\longrightarrow}-\frac{\mathrm{i}}{\hbar}\left[\widehat{\overline{\mathrm{f}}^{\alpha}(F)}, \widehat{\overline{\mathrm{f}}_{\alpha}(G)}\right]=-\frac{\mathrm{i}}{\hbar}\left[\overline{\mathrm{f}}^{\alpha}(\hat{F}), \overline{\mathrm{f}}_{\alpha}(\hat{G})\right]=-\frac{\mathrm{i}}{\hbar}[\hat{F}, \hat{G}]_{\star}
$$

The second equality holds because the lifts $P_{i}$ and $\hat{P}_{i}$ of $\frac{\partial}{\partial x^{i}}$ satisfy $\left\{\widehat{P_{i}, G}\right\}=-\frac{\mathrm{i}}{\hbar}\left[\widehat{P}_{i}, \widehat{G}\right]$ at leading order in $\hbar$.

Repeating the passages of (5.15) we obtain (in accordance with (5.25)) the $\star$-commutator of the fields $\hat{\Phi}$ and $\hat{\Pi}$,

$$
[\hat{\Phi}(x), \hat{\Pi}(y)]_{\star}=i \hbar \delta(x-y) .
$$

Concerning the creation and annihilation operators, they are functionals of the operators $\hat{\Phi}, \hat{\Pi}$ through the quantum analogue of the classical functional relation (5.17). Their $\star$-commutator follows from (5.26)

$$
\left[\hat{a}(k), \hat{a}^{\dagger}\left(k^{\prime}\right)\right]_{\star}=(2 \pi)^{d} \delta\left(k-k^{\prime}\right) .
$$


We have derived this relation from our quantization scheme for field theories on noncommutative space dictated by the symmetry Hopf algebras $U \mathrm{~A}_{\star}$ and $U \hat{\mathrm{A}}_{\star}$ and their deformed Lie brackets. In order to compare this expression with similar ones which have been found in the literature [26, 27, 28, 29, 30, 33, 34, 35] it is useful to recall (5.22) and realize the action of the $\mathcal{R}$-matrix. Since $\mathcal{R}=\mathcal{F}^{-2}$ we obtain that (5.27) is equivalent to

$$
\hat{a}(k) \star \hat{a}^{\dagger}\left(k^{\prime}\right)-\mathrm{e}^{-\mathrm{i} \theta^{i j} k_{i}^{\prime} k_{j}} \hat{a}^{\dagger}\left(k^{\prime}\right) \star \hat{a}(k)=(2 \pi)^{d} \delta\left(k-k^{\prime}\right) .
$$

This relation first appeared in [37]. In the noncommutative QFT context it appears in [34], [33], and implicitly in [32], it is also contemplated in [35] as a second option. On the other hand [27, 28, 29, 35], starting from a different definition of $\star$-commutator, $[A \star B]:=$ $A \star B-B \star A$, obtain deformed commutation relations of the kind $a_{k} a_{k^{\prime}}^{\dagger}-\mathrm{e}^{-\frac{i}{2} \theta^{i j} k_{i} k_{j}^{\prime}} a_{k^{\prime}}^{\dagger} a_{k}=$ $(2 \pi)^{d} \delta\left(k-k^{\prime}\right)$. These are different from (5.28), indeed if we expand also the $\star$-product in (5.28) we obtain the usual commutation relations $\hat{a}(k) \hat{a}^{\dagger}\left(k^{\prime}\right)-\hat{a}^{\dagger}\left(k^{\prime}\right) \hat{a}(k)=(2 \pi)^{d} \delta\left(k-k^{\prime}\right)$.

As in the case of the $\star$-Poisson bracket, we have found that the $\star$-commutator of coordinate fields (5.26), and of creation and annihilation operators (5.27), are equal to the usual undeformed ones. Once again, we warn the reader that this is not true anymore

for more complicated functionals of the coordinate fields, in general $[\hat{F}, \hat{G}]_{\star} \neq[\hat{F}, \hat{G}]$.

\section{Acknowledgments}

I felt very honored to present this work at the conference "Geometry and Operators Theory" in celebration of the 65th birthday of Nicola Teleman. This research is based on joint work with Marija Dimitriević, Frank Meyer, Julius Wess and with Fedele Lizzi, Patrizia Vitale; I would like to thank them for the fruitful collaboration. Partial support from the European Community's Human Potential Program under contract MRTN-CT2004-005104 and the Italian MIUR under contract PRIN-2005023102 is acknowledged.

\section{References}

[1] S. Doplicher, K. Fredenhagen and J. E. Roberts, "The Quantum structure of spacetime at the Planck scale and quantum fields," Commun. Math. Phys. 172 (1995) 187 arXiv:hep-th/0303037.

[2] S. Doplicher, "Spacetime and fields, a quantum texture," arXiv:hep-th/0105251.

[3] A. Connes, Noncommutative Geometry, Academic Press, (1994).

[4] G. Landi, An introduction to noncommutative spaces and their geometry, Springer, (1997). arXiv:hep-th/9701078. 
[5] J. M. Gracia-Bondia, J. C. Varilly and H. Figueroa, Elements Of Noncommutative Geometry, Birkhaeuser (2001)

[6] W. Heisenberg Letter from Heisenberg to Peierls in: W. Pauli, Scientific Correspondence, Vol II Berlin, Springer (1985).

[7] V. G. Drinfeld, "On constant quasiclassical solutions of the Yang-Baxter equations", Soviet Math. Dokl. 28 (1983) 667-671;

[8] A. Connes and G. Landi, Noncommutative manifolds: The instanton algebra and isospectral deformations, Commun. Math. Phys. 221, 141 (2001) math.qa/0011194.

[9] A. Connes and M. Dubois-Violette, Noncommutative finite-dimensional manifolds. I. Spherical manifolds and related examples, math.qa/0107070.

[10] V. Gayral, B. Iochum and J. C. Varilly, Dixmier traces on noncompact isospectral deformations, hep-th/0507206.

[11] J. C. Varilly, Quantum symmetry groups of noncommutative spheres, Commun. Math. Phys. 221 (2001) 511 math.qa/0102065.

[12] A. Sitarz, Twists and spectral triples for isospectral deformations, Lett. Math. Phys. 58 (2001) 69-79, math.QA/0102074, Rieffel's deformation quantization and isospectral deformations. Int. J. Theor. Phys. 40 (2001) 1693. math.QA/0102075.

[13] P. Aschieri and F. Bonechi, On the Noncommutative Geometry of Twisted Spheres, Lett. Math. Phys. 59 (2002) 133 math.qa/0108136.

[14] V. G. Drinfeld, "Quasi-Hopf Algebras" Leningrad Math. J. 1 (1990) 1419 [Alg. Anal. 1N6 (1989) 114].

[15] P. Aschieri, C. Blohmann, M. Dimitrijevic, F. Meyer, P. Schupp and J. Wess, "A gravity theory on noncommutative spaces," Class. Quant. Grav. 22, (2005) 3511 arXiv:hep-th/0504183.

[16] P. Aschieri, M. Dimitrijevic, F. Meyer and J. Wess, "Noncommutative geometry and gravity," Class. Quant. Grav. 23, (2006) 1883 [arXiv:hep-th/0510059].

[17] P. Aschieri, "Noncommutative symmetries and gravity," J. Phys. Conf. Ser. 53, 799 (2006) arXiv:hep-th/0608172]; "Noncommutative gravity and the *-Lie algebra of diffeomorphisms," Fortsch. Phys. 55 (2007) 649 [arXiv:hep-th/0703014].

[18] P. Aschieri, F. Lizzi and P. Vitale, "Twisting all the way: from Classical Mechanics to Quantum Fields," Phys. Rev. D 77 (2008) 025037 [arXiv:0708.3002 [hep-th]]. 
[19] M. Gerstenhaber, A. Giaquinto, S. D. Schack, in Quantum symmetry. Proceedings in EIMI 1990, Lect. Notes Math. 1510 ed. P. P. Kulish (Springer-Verlag, Berlin, 1992) pp. $9-46$.

[20] O. V. Ogievetsky, in Proc. Winter School Geometry and Physics, Zidkov, Suppl. Rendiconti cir. Math. Palermo, Serie II - N 37, 185 (1993); preprint MPI-Ph/92-99, Munich, (1992) 14p.

[21] P.P. Kulish, V.D. Lyakhovsky, A.I. Mudrov Extended jordanian twists for Lie algebras, J. Math. Phys. 40, 4569 (1999), math.QA/9806014,

P.P. Kulish, V.D. Lyakhovsky and M.A. del Olmo, Chains of twists for classical Lie algebras, J. Phys. A: Math. Gen. 32, 8671 (1999), math.QA/9908061.

[22] D. Gurevich and S. Majid, Braided groups of Hopf algebras obtained by twisting, Pac. J. Math. 162:27-44, 1994.

[23] S. L. Woronowicz, "Differential calculus on compact matrix pseudogroups (quantum groups)," Commun. Math. Phys. 122, 125 (1989).

[24] P. Aschieri, "Lectures on Hopf algebras, quantum groups and twists," proceedings of the Second Modave Summer School In Mathematical Physics, International Solvay Intitutes, 208-219, arXiv:hep-th/0703013.

P. Aschieri, M. Dimitrijevic, P. Kulish, F. Lizzi, J. Wess, "Noncommutative Spacetimes," Lecture Notes in Physics, Vol. 774 ISBN: 978-3-540-89792-7, Springer (to appear). Chapter 7.

[25] P. Aschieri et al. In preparation.

[26] G. Fiore and P. Schupp, "Statistics and Quantum Group Symmetries," arXiv:hep-th/9605133|; "Identical particles and quantum symmetries," Nucl. Phys. B 470, (1996) 211 [arXiv:hep-th/9508047].

[27] A. P. Balachandran, T. R. Govindarajan, G. Mangano, A. Pinzul, B. A. Qureshi and S. Vaidya, "Statistics and UV-IR mixing with twisted Poincare invariance," Phys. Rev. D 75 (2007) 045009 arXiv:hep-th/0608179.

[28] A. P. Balachandran, G. Mangano, A. Pinzul and S. Vaidya, "Spin and statistics on the Groenwald-Moyal plane: Pauli-forbidden levels and transitions," Int. J. Mod. Phys. A 21 (2006) 3111 arXiv:hep-th/0508002.

[29] F. Lizzi, S. Vaidya and P. Vitale, "Twisted conformal symmetry in noncommutative two-dimensional quantum field theory," Phys. Rev. D 73 (2006) 125020 arXiv:hep-th/0601056. 
[30] A. Tureanu, "Twist and spin-statistics relation in noncommutative quantum field theory," Phys. Lett. B 638 (2006) 296 arXiv:hep-th/0603219.

[31] P. G. Castro, B. Chakraborty and F. Toppan, "Wigner Oscillators, Twisted Hopf Algebras and Second Quantization," J. Math. Phys. 49 (2008) 082106 arXiv:0804.2936 [hep-th]].

[32] J. Zahn, "Remarks on twisted noncommutative quantum field theory," Phys. Rev. D 73, 105005 (2006) arXiv:hep-th/0603231.

[33] J. G. Bu, H. C. Kim, Y. Lee, C. H. Vac and J. H. Yee, "Noncommutative field theory from twisted Fock space," Phys. Rev. D 73 (2006) 125001 |arXiv:hep-th/0603251].

[34] P.P. Kulish "Twist of quantum groups and noncommutative field theory" arXiv:hep-th/0606056.

[35] G. Fiore and J. Wess, "On 'full' twisted Poincare symmetry and QFT on Moyal-Weyl spaces," Phys. Rev. D 75 (2007) 105022 arXiv:hep-th/0701078].

[36] J. Wess, "Deformed gauge theories," J. Phys. Conf. Ser. 53, 752 (2006) arXiv:hep-th/0608135.

[37] H. Grosse , "On the construction of Möller operators for the nonlinear Schrödinger equation," Physics Letters B 86 267-271 (1979).

A. B. Zamolodchikov and A. B. Zamolodchikov, "Factorized S-matrices in two dimensions as the exact solutions of certain relativistic quantum field models," Annals Phys. 120 253-291 (1979).

L. D. Faddeev, "Quantum completely integral models of field theory," Sov. Sci. Rev. C1 107-155 (1980). 